\title{
Progressive Change in the Form of Growth Gradients
}

\section{Growth Gradients in the Abdominal LimbS OF THE SHORE-CRAB}

\section{By Prof. J. S. Huxley}

$\mathrm{T}$ HE re-analysis of Shen's data (1935) on early post-larval development in Carcinus monas has proved interesting in showing a graded change with time in the shape of the growth gradients of the abdominal appendages. Dr. J. H. Day's work on the abdominal segments of the same species (see below) has led to similar conclusions. Since the phenomenon has not previously been recorded, at least in quantitative terms, it was felt advisable to publish simultaneous notes on the subject.

Each point in the graphs in Figs. 1 and 2 represents a single individual, so that the results cannot pretend to such accuracy as Day's. None the less, the general results are clear. Two main phases aro involved: (1) a chiefly regressive phase, during which the large Megalopa limbs are converted into the postlarval type; (2) a progressive phase, during which those limbs represented in the adult show varying degrees of high positive heterogony. At the 5th or 6 th instar, there is evidence of the onset of a third phase, of stabilisation (and we may presume that sexual maturity will introduce yet a fourth growth phase). Growth-coefficients $(k)$ have been calculated according to the formula $y=b x^{k}$ : carapace breadth has been used as standard $(x)$. When, as during the regressive phase, a part diminishes in absolute size, the growth-coefficients have been given a negative sign : these cannot have the same biological significance as ordinary growth-coefficients, but serve to show the relative intensity of regression.

In males (Fig. 1) the regressive phase becomes more active in stage $B$ than at the metamorphosis (A), except that the first abdominal (first copulatory)

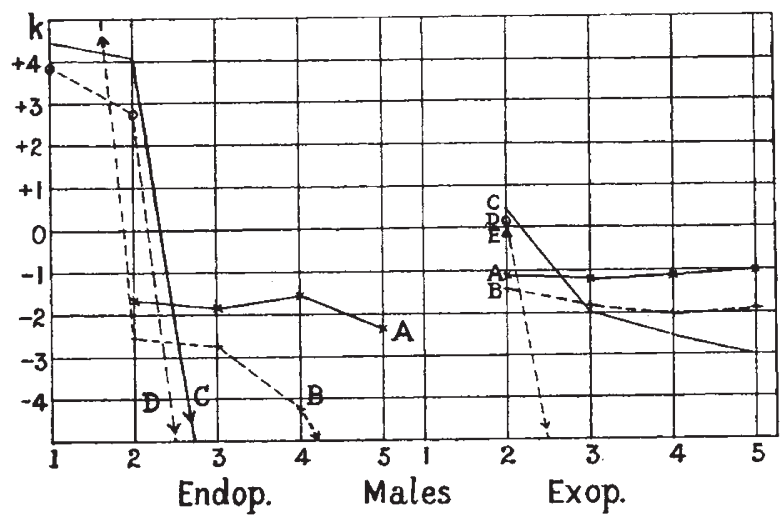

FIG. 1. Growth-gradients of endopodites and exopodites of the abdominal limbs of male Carcinus manas in successive stages. Ordinates, growth-coefficients $(k)$; abscissæ, abdominal limbs. Stages : $A$, metamorphosis (megalopa to 1 st young crab instar); $B$, 1st-2nd instar; $C$, 3rd-4th; $D$, 4th-5th; $E$ (shown for exopodites only) 5th-6th. Arrows indicate growth-coefficients of, or approximating to, infinity.

appendage, represented by endopodite only, first appears then. The second copulatory appendage does not begin positive growth until the next moult. The regressive gradient after stage $A$ has its high point posteriorly; the later positive gradient of appendages 1 and 2 has its high point anteriorly. The interaction of the two gradients is clearly shown, notably by the exopodites. These are all destined to disappear ; but owing to the presence of the region of high growth in limbs 1 and 2, the regressive gradient in stage $C$ is tilted upwards anteriorly;

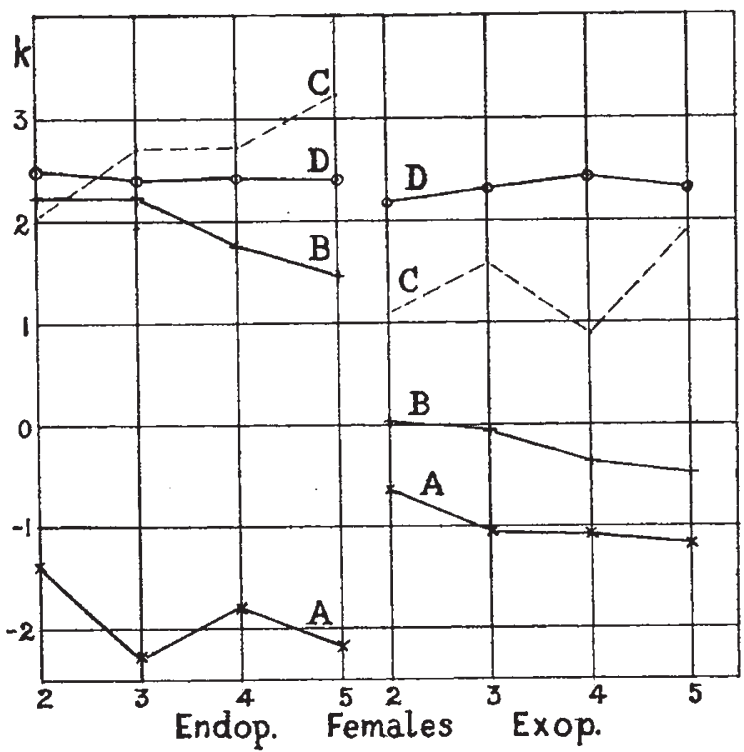

FIG 2. Similar to Fig. 1 , but for females. Stages: $A$, metamorphosis (as in male); $B$, 1st-3rd instar ; $C$, 3rd-5th; $D, 5$ th-7th.

exopodite 2 actually acquires positive growthintensity in stage $C$, and in subsequent stages only loses it gradually. We can say that it is made to postpone its disappearance by becoming temporarily infected by the high positive growth-intensity in the endopodite and in limb 1. The intensity of growthchange, whether regressive or positive, is greater in each phase in the endopodites.

In females (Fig. 2), this last rule also holds. The tendency for regression to be more intense posteriorly is also obvious. But during the succeeding phase of positive heterogony, the high point is posterior (clearly shown in endopodites, stage $C$ ), perhaps in correlation with the similar-shaped gradient in abdominal segments (see Day's note). The gradient for the next stage (stability) is practically horizontal.

To sum up, both sexes show evidence of different growth-gradients succeeding each other in the same region, and the changes in growth-gradients are themselves graded. The changes in the female might be produced by a mere change of sign, but in the male a new growth-centre clearly originates in the anterior region, confirming Day's suggestion. Any changes are usually shown at an earlier stage by the high point of a gradient than by its lower portions. In addition, there is always a medio-lateral growthgradient, shown by the fact that the growth-changes in the endopodites are always more intense (and are initiated earlier). 